\title{
Extremal infinite overlap-free binary words
}

\author{
Jean-Paul Allouche \\ CNRS, LRI \\ Bâtiment 490 \\ F-91405 Orsay Cedex (France) \\ allouche@lri.fr \\ James Currie \\ Department of Mathematics \\ University of Winnipeg \\ Winnipeg, Manitoba R3B 2E9 (Canada) \\ currie@io.uwinnipeg.ca \\ Jeffrey Shallit \\ Department of Computer Science \\ University of Waterloo \\ Waterloo, Ontario N2L 3G1 (Canada) \\ shallit@graceland.uwaterloo.ca
}

Submitted: August 29, 1997; Accepted: May 3, 1998.

\begin{abstract}
Let $\overline{\mathbf{t}}$ be the infinite fixed point, starting with 1 , of the morphism $\mu: 0 \rightarrow 01$, $1 \rightarrow 10$. An infinite word over $\{0,1\}$ is said to be overlap-free if it contains no factor of the form axaxa, where $a \in\{0,1\}$ and $x \in\{0,1\}^{*}$. We prove that the lexicographically least infinite overlap-free binary word beginning with any specified prefix, if it exists, has a suffix which is a suffix of $\overline{\mathbf{t}}$. In particular, the lexicographically least infinite overlap-free binary word is $001001 \overline{\mathbf{t}}$.
\end{abstract}

Keywords: Homomorphism, fixed point, overlap-free word. 1991 Mathematics Subject Classification: Primary 68R15. 


\section{Introduction}

Since the pioneering work of Thue $[14,15]$ (see also [5]) the overlap-free words on a finite alphabet, i.e., those words that do not contain a factor axaxa, where $x$ is a word and $a$ a letter, have been studied extensively. The question of extremality (for the lexicographic order) of overlap-free binary infinite words seems to have been addressed only once: Berstel proved [4], (see also [5]), that the lexicographically greatest infinite overlap-free word on the binary alphabet $\{0,1\}$ that begins with 0 is the Thue-Morse sequence $\mathbf{t}=01101001 \cdots$, which shows once more the ubiquity of this sequence. (Recall that $\mathbf{t}$ is one of the fixed points of the morphism $0 \longrightarrow 01,1 \longrightarrow 10$. We let $\overline{\mathbf{t}}=10010110 \cdots$ denote the other fixed point.) The following natural question then arises: what is the lexicographically least overlap-free word on $\{0,1\}$ that begins with 0 ? Computing the first few terms suggests that this word is $0010011001011001101001 \cdots=001001 \overline{\mathbf{t}}$.

The main result of this paper is the following: the lexicographically least overlap-free sequence with any specified prefix, if it exists, has a suffix equal to a suffix of $\overline{\mathbf{t}}$. Furthermore, we give an algorithm to construct this sequence. Of course, replacing "least" by "greatest" and interchanging 0's and 1's gives a dual result.

\section{Notation}

In what follows we consider words or infinite words (sequences) on the binary alphabet $\{0,1\}$. Words will be denoted by lower-case letters (usually $r, s, \cdots$ ). The set of all finite words on $\{0,1\}$ is denoted by $\{0,1\}^{*}$. Elements of $\{0,1\}$ will be denoted by $a, b, c, d, \cdots$. Infinite words will be denoted by boldface small letters $\mathbf{x}, \mathbf{y}, \cdots$. The length (number of letters) of a (finite) word $w$ is denoted by $|w|$. If $w$ is a word, $\bar{w}$ is the word obtained from $w$ by replacing the 0 's by 1 's and the 1 's by 0 's.

We define the morphism $\mu$ on $\{0,1\}^{*}$ by $\mu(0)=01, \mu(1)=10$, and we extend it to infinite words by continuity. The infinite fixed point of $\mu$ beginning with 0 is denoted by $\mathbf{t}$, hence

$$
\mathbf{t}=0110100110010110 \cdots
$$

The infinite fixed point of $\mu$ beginning with 1 is $\overline{\mathbf{t}}$. These sequences are called the Thue-Morse sequences.

The lexicographic order for infinite words is defined by $\mathbf{x}<\mathbf{y}$ if and only if there exists $k \geq 0$ such that the prefixes of length $k$ of $\mathbf{x}$ and $\mathbf{y}$ are equal, and the $(k+1)$-th letter of $\mathbf{x}$ is 0 while the $(k+1)$-th letter of $\mathbf{y}$ is 1 . We note that the morphism $\mu$ is order-preserving on the set of infinite words; more precisely, $\mathbf{x}<\mathbf{y} \Leftrightarrow \mu(\mathbf{x})<\mu(\mathbf{y})$.

\section{Tools}

In this section we provide some basic tools that will be useful in the proof of our main result. The three lemmas we give demonstrate the close relationship between the morphism $\mu$ and overlap-free words. Parts of the lemmas below can be found in the literature (essentially in 
$[14,15]$; see also [6]). Nevertheless, it seems that in the factorization lemma we give below (Lemma 3), the question of uniqueness of the decomposition was first addressed in [11].

We start with a technical lemma.

\section{Lemma 1}

1. If $y, y^{\prime} \in\{0,1\}^{*}$, and if there exist $c, d \in\{0,1\}$ such that $u=c \mu(y)=\mu\left(y^{\prime}\right) d$, then $u=c(\bar{c} c)^{|y|}$.

2. If $y, z \in\{0,1\}^{*}$ satisfy $z z=\mu(y)$, then there exists $x \in\{0,1\}^{*}$ such that $z=\mu(x)$.

\section{Proof.}

1. We first note that $y$ and $y^{\prime}$ must have the same length. Let $y=a_{1} a_{2} \cdots a_{s}$ and $y^{\prime}=b_{1} b_{2} \cdots b_{s}$. Then we have:

$$
c a_{1} \overline{a_{1}} a_{2} \overline{a_{2}} \cdots a_{s-1} \overline{a_{s-1}} a_{s} \overline{a_{s}}=b_{1} \overline{b_{1}} b_{2} \overline{b_{2}} \cdots b_{s-1} \overline{b_{s-1}} b_{s} \overline{b_{s}} d .
$$

Hence $b_{1}=c, a_{1}=\overline{b_{1}}=\bar{c}, b_{2}=\overline{a_{1}}=c, a_{2}=\overline{b_{2}}=\bar{c}, \cdots, b_{s}=\overline{a_{s-1}}=c, a_{s}=\overline{b_{s}}=\bar{c}$, and $\overline{a_{s}}=d$, i.e., $c=d$, and hence finally $u=c(\bar{c} c)^{s}$.

2. Suppose that $z z=\mu(y)$. If $|z|$ is even, the result is clear, since then $|\mu(y)| \equiv 0(\bmod 4)$, and hence $|y|$ is even. Hence $z$ is the image by $\mu$ of the prefix of $y$ of length $|y| / 2$. Let us show that $|z|$ cannot be odd. If it were, let $z=a u=v b$, where $a, b \in\{0,1\}$ and $u$ and $v$ are binary words of even length. Then $\mu(y)=z z=v b a u$; hence $u$ and $v$ must be the images by $\mu$ of binary words, say $u=\mu(r)$ and $v=\mu(s)$. Hence $z z=\mu(s) b a \mu(r)$, and $b=\bar{a}$. But we have $z=a \mu(r)=\mu(s) b$, hence from assertion 1 above, $z=a(\bar{a} a)^{|r|}$. But the last letter of $z$ cannot be simultaneously equal to $a$ and to $\bar{a}$.

Next, we prove that the morphism $\mu$ behaves nicely when applied to overlap-free words.

\section{Lemma 2}

1. Let $w \in\{0,1\}^{*}$. Then $w$ is overlap-free if and only if $\mu(w)$ is overlap-free.

2. Let $\mathbf{x}$ be an infinite word on $\{0,1\}$. Then

(a) $\mu(\mathbf{x})$ is overlap-free if and only if $\mathbf{x}$ is overlap-free.

(b) $0 \mu(\mathbf{x})$ is overlap-free if and only if $1 \mathbf{x}$ is overlap-free.

(c) $1 \mu(\mathbf{x})$ is overlap-free if and only if $0 \mathbf{x}$ is overlap-free.

(d) $00 \mu(\mathbf{x})$ is overlap-free if and only if $1 \mathbf{x}$ is overlap-free and $\mathbf{x}$ begins with 101.

(e) $11 \mu(\mathbf{x})$ is overlap-free if and only if $0 \mathbf{x}$ is overlap-free and $\mathbf{x}$ begins with 010 .

3. The Thue-Morse sequences $\mathbf{t}$ and $\overline{\mathbf{t}}$ are overlap-free. The sequences $0 \mathbf{t}, 1 \mathbf{t}, 0 \overline{\mathbf{t}}$, and $1 \overline{\mathbf{t}}$ are overlap-free.

4. The sequences $01 \overline{\mathbf{t}}, 10 \overline{\mathbf{t}}, 110 \overline{\mathbf{t}}$, and $001001 \overline{\mathbf{t}}$ are overlap-free. 


\section{Proof.}

1. This is proved in [15].

2. (a) As in [15], assertion 1 above immediately extends to infinite words, showing that $\mu(\mathbf{x})$ is overlap-free if and only if $\mathbf{x}$ is overlap-free.

(b) Now if the infinite word $0 \mu(\mathbf{x})$ contains an overlap, then the word $\mathbf{s}=10 \mu(\mathbf{x})$ a fortiori contains an overlap. Since $\mathbf{s}=\mu(1 \mathbf{x})$, this implies that $1 \mathbf{x}$ contains an overlap. If conversely the word $1 \mathbf{x}$ contains an overlap, it can occur in two ways: either the overlap is a prefix of $1 \mathbf{x}$, hence $\mathbf{x}$ begins with $z 1 z 1$ for some finite word $z$, or the overlap occurs "inside" $1 \mathbf{x}$, which means that $\mathbf{x}$ itself contains an overlap. In the latter case, $\mu(\mathbf{x})$ contains an overlap, hence $0 \mu(\mathbf{x})$ contains an overlap. In the former case, $0 \mu(\mathbf{x})$ begins with $0 \mu(z) 10 \mu(z) 10$ and hence contains an overlap. This proves (b). The proof of (c) is similar.

(d) Suppose now that $00 \mu(\mathbf{x})$ is overlap-free. Then $0 \mu(\mathbf{x})$ is also overlap-free; hence, from the preceding argument, $1 \mathbf{x}$ is overlap-free. Furthermore, if $\mathbf{x}$ begins with $a b c$ where $a, b, c \in\{0,1\}$, then $00 a \bar{a} b \bar{b} c \bar{c}$ and $1 a b c$ must be overlap-free; hence, easily, $a=1, b=0$, and $c=1$. Conversely, suppose that $1 \mathbf{x}$ is overlap-free and begins with 101 . From the preceding argument this implies that $0 \mu(\mathbf{x})$ is overlap-free. Hence any overlap of the word $00 \mu(\mathbf{x})$ must in fact be a prefix. Hence, in order to show that $00 \mu(\mathbf{x})$ is overlap-free, we will suppose that $00 \mu(\mathbf{x})$ begins with azaza, with $a \in\{0,1\}$ and $z \in\{0,1\}^{*}$ and obtain a contradiction. By the hypothesis, the word $\mathbf{x}$ begins with 101 , say $\mathbf{x}=101 \mathbf{y}$ for some infinite word $\mathbf{y}$, hence the word $00 \mu(\mathbf{x})$ is equal to $00100110 \mu(\mathbf{y})$, and begins with azaza. By inspection, we see that $|a z| \geq 6$, hence the word 001001 occurs as a factor of $10 \mu(\mathbf{y})$. Since this last word begins with 1 , this means that there exists a nonempty word $w$ such that $10 \mu(\mathbf{y})=w 001001 \cdots$. But $10 \mu(\mathbf{y})$ is overlap-free, as it is a factor of the overlap-free word $0 \mu(\mathbf{x})$. This implies that 001001 can be extended to the left (with the last letter of $w$ ) to an overlap-free word, although 001001 is clearly not extendable to the left to an overlap-free word. This proves (d). The proof of (e) is similar.

3. The Thue-Morse sequence $\overline{\mathbf{t}}$ is the limit, as $n \rightarrow \infty$, of the binary words $\mu^{n}(1)$ which are all overlap-free, since 1 is overlap-free: this is Thue's proof [15].

Let $a \in\{0,1\}$, and suppose that $a \overline{\mathbf{t}}$ contains an overlap. Then the overlap must be a prefix of $a \overline{\mathbf{t}}$. Hence there exists a finite word $z$ such that $\overline{\mathbf{t}}$ begins with $z a z a$. We will show that this is impossible. More precisely we show that the sequence $\overline{\mathbf{t}}$ cannot begin with $z 0 z 0$ nor $z 1 z 1$ for any finite word $z$. Let us suppose that $\overline{\mathbf{t}}$ begins with $z a z a$ with $z$ a finite word and $a \in\{0,1\}$, and take $z$ a word of minimal length for which there exists $a \in\{0,1\}$ such that $\overline{\mathbf{t}}$ begins with $z a z a$. Since $z a z a$ has even length and $\overline{\mathbf{t}}=\mu(\overline{\mathbf{t}})$, the word $z a z a$ is the image by $\mu$ of a binary word. Hence, applying Lemma 1, the word $z a$ itself is the image of a binary word by $\mu$, say $z a=\mu(y)$. Hence $y$ must end with $\bar{a}$, say $y=x \bar{a}$. Then $\overline{\mathbf{t}}$ begins with $z a z a=\mu(x \bar{a} x \bar{a})$. But $\overline{\mathbf{t}}=\mu(\overline{\mathbf{t}})$. Hence $\overline{\mathbf{t}}$ begins with $x \bar{a} x \bar{a}$, which contradicts the minimality of the length of $z$.

4. Since $01 \overline{\mathbf{t}}=\mu(0 \overline{\mathbf{t}})$, and $10 \overline{\mathbf{t}}=\mu(1 \overline{\mathbf{t}})$, we deduce from 2.(a) and 3 above that these two words are overlap-free. The word $110 \overline{\mathbf{t}}$ is overlap-free, since it is a suffix of the word 
$0110 \overline{\mathbf{t}}=\mu^{2}(0 \overline{\mathbf{t}})$ that is overlap-free from 2.(a) and 3 above. Finally to prove that the word $001001 \overline{\mathbf{t}}$ is overlap-free, we write $001001 \overline{\mathbf{t}}=00 \mu(10 \overline{\mathbf{t}})$. This last word is overlap-free from (d) above, since we have just proved that $110 \overline{\mathbf{t}}$ is overlap-free.

We now give a factorization lemma for both finite and infinite overlap-free binary words.

\section{Lemma 3}

1. If $x \in\{0,1\}^{*}$ is overlap-free, then there exist $u, v, y$ with $u, v \in\{\varepsilon, 0,1,00,11\}$ and $y \in\{0,1\}^{*}$ an overlap-free word, such that $x=u \mu(y) v$. Furthermore this decomposition is unique if $|x| \geq 7$, and $u$ (resp. $v$ ) is completely determined by the prefix (resp. suffix) of length 7 of $x$. The bound 7 is sharp as shown by the example $x=001011=00 \mu(1) 11=0 \mu(00) 1$.

2. If $\mathbf{x}$ is an infinite overlap-free word on $\{0,1\}$, then there exist $u \in\{\varepsilon, 0,1,00,11\}$ and an infinite overlap-free word $\mathbf{y}$ on $\{0,1\}$ such that $\mathbf{x}=u \mu(\mathbf{y})$. The prefix $u$ is completely determined by the prefix of $\mathbf{x}$ of length 4 , except if $\mathbf{x}$ begins with 0010 or 1101, in which case the word $u$ is completely determined by the prefix of $\mathbf{x}$ of length 5 .

Proof. First note that the word $y$ is necessarily overlap-free by assertion 1 of Lemma 2.

The existence of the decomposition for finite words is proved in [10], in the proof of Theorem 6.4. The uniqueness is proved in [11], Lemma 2.2.

Finally in this factorization of $x$, the word $u$ (resp. $v$ ) depends only on the prefix (resp. suffix) of $x$ of length 7 . This is recalled in the following table of all possible prefixes (resp. suffixes) of length $\geq 7$ : by mere inspection, we see that the word $u$ (resp. $v$ ) is uniquely determined, knowing the decomposition does exist. (Note however that some of the words below, e.g., 0011011, might be non-extendable to longer overlap-free words.)

\begin{tabular}{|c|c|}
\hline$x$ & $u$ \\
\hline $0010011 \cdots$ & 00 \\
$0010110 \cdots$ & 0 \\
$0011001 \cdots$ & 0 \\
$0011010 \cdots$ & 0 \\
$0011011 \cdots$ & 0 \\
$0100101 \cdots$ & 0 \\
\hline
\end{tabular}

\begin{tabular}{|c|c|}
\hline$x$ & $u$ \\
\hline $0100110 \cdots$ & 0 \\
$0101100 \cdots$ & $\varepsilon$ \\
$0101101 \cdots$ & $\varepsilon$ \\
$0110010 \cdots$ & $\varepsilon$ \\
$0110011 \cdots$ & $\varepsilon$ \\
$0110100 \cdots$ & $\varepsilon$ \\
\hline
\end{tabular}

\begin{tabular}{|c|c|}
\hline$x$ & $u$ \\
\hline $1001011 \cdots$ & $\varepsilon$ \\
$1001100 \cdots$ & $\varepsilon$ \\
$1001101 \cdots$ & $\varepsilon$ \\
$1010010 \cdots$ & $\varepsilon$ \\
$1010011 \cdots$ & $\varepsilon$ \\
$1011001 \cdots$ & 1 \\
\hline
\end{tabular}

\begin{tabular}{|c|c|}
\hline$x$ & $u$ \\
\hline $1011010 \cdots$ & 1 \\
$1100100 \cdots$ & 1 \\
$1100101 \cdots$ & 1 \\
$1100110 \cdots$ & 1 \\
$1101001 \cdots$ & 1 \\
$1101100 \cdots$ & 11 \\
\hline
\end{tabular}

\begin{tabular}{|c|c|}
\hline$x$ & $v$ \\
\hline$\cdots 0010011$ & 1 \\
$\cdots 0010110$ & $\varepsilon$ \\
$\cdots 0011001$ & $\varepsilon$ \\
$\cdots 0011010$ & $\varepsilon$ \\
$\cdots 0011011$ & 11 \\
$\cdots 0100101$ & $\varepsilon$ \\
\hline
\end{tabular}

\begin{tabular}{|c|c|}
\hline$x$ & $v$ \\
\hline$\cdots 0100110$ & $\varepsilon$ \\
$\cdots 0101100$ & 0 \\
$\cdots 0101101$ & 1 \\
$\cdots 0110010$ & 0 \\
$\cdots 0110011$ & 1 \\
$\cdots 0110100$ & 0 \\
\hline
\end{tabular}

\begin{tabular}{|c|c|}
\hline$x$ & $v$ \\
\hline$\cdots 1001011$ & 1 \\
$\cdots 1001100$ & 0 \\
$\cdots 1001101$ & 1 \\
$\cdots 1010010$ & 0 \\
$\cdots 1010011$ & 1 \\
$\cdots 1011001$ & $\varepsilon$ \\
\hline
\end{tabular}

\begin{tabular}{|c|c|}
\hline$x$ & $v$ \\
\hline$\cdots 1011010$ & $\varepsilon$ \\
$\cdots 1100100$ & 00 \\
$\cdots 1100101$ & $\varepsilon$ \\
$\cdots 1100110$ & $\varepsilon$ \\
$\cdots 1101001$ & $\varepsilon$ \\
$\cdots 1101100$ & 0 \\
\hline
\end{tabular}


2. For any prefix $x_{n}$ of $\mathbf{x}$ such that, say $\left|x_{n}\right|=n$, assertion 1 of Lemma 3 above gives the existence of $u_{n}$ and $v_{n}$ in $\{\varepsilon, 0,1,00,11\}$ and of an overlap-free word $y_{n}$ on $\{0,1\}$, such that $x_{n}=u_{n} \mu\left(y_{n}\right) v_{n}$. Furthermore, $u_{n}$ does not depend on $n$ for $n \geq 7$. Say $u_{n}=u$. Hence $\mathbf{x}=$ $\lim _{n \rightarrow \infty} x_{n}=\lim _{n \rightarrow \infty} u \mu\left(y_{n}\right) v_{n}$. Since $\mu\left(y_{n}\right)$ goes to infinity, this implies $\mathbf{x}=\lim _{n \rightarrow \infty} u \mu\left(y_{n}\right)$. Hence $\lim _{n \rightarrow \infty} \mu\left(y_{n}\right)$ exists, which gives the existence of $\mathbf{y}=\lim _{n \rightarrow \infty} y_{n}$. This sequence is overlap-free as it is a limit of overlap-free words, and we have $\mathbf{x}=u \mu(\mathbf{y})$. Note that in the decomposition of $x_{n}, v_{n}$ cannot be equal to 00 or 11 , since these words are not images of a word by $\mu$. Hence $v_{n}$ is either empty or is equal to 0 or 1 . Finally, inspecting the table above shows that the prefix of length 4 of a 7-letter word determines the word $u$ in all cases but the two cases where the word begins with 0010 or 1101 for which we need to look at the prefix of length 5.

\section{The main result}

Before proving our main theorem, we need to state two results on overlap-free sequences and to study eight particular cases. This is the purpose of the following proposition.

Proposition 1 Let $w$ be a binary word, and let $\mathbf{x}(w)$ be the lexicographically least overlapfree sequence (if it exists) beginning with $w$. Let $\overline{\mathbf{t}}$ be the Thue-Morse sequence beginning with 1. Then

(a) $\mathbf{x}(1)=\overline{\mathbf{t}}$.

(b) If $\mathbf{x}(w)$ exists, and if $z$ is a finite word such that $z \mathbf{x}(w)$ is overlap-free, then $\mathbf{x}(z w)$ exists and $\mathbf{x}(z w)=z \mathbf{x}(w)$. In particular $\mathbf{x}(01)=0 \overline{\mathbf{t}}$ and $\mathbf{x}(11)=1 \overline{\mathbf{t}}$.

If $\mathbf{x}(w)$ exists, and if $w w_{1}$ is a prefix of $\mathbf{x}(w)$, then $\mathbf{x}\left(w w_{1}\right)$ exists and $\mathbf{x}\left(w w_{1}\right)=\mathbf{x}(w)$. In particular $\mathbf{x}(10)=\overline{\mathbf{t}}$.

(c) We have $\mathbf{x}(001)=001001 \overline{\mathbf{t}}$. Furthermore, $\mathbf{x}(0)=\mathbf{x}(00)=001001 \overline{\mathbf{t}}$.

(d) $\mathbf{x}(010)=0 \overline{\mathbf{t}}$.

(e) $\mathbf{x}(011)=01 \overline{\mathbf{t}}$.

(f) $\mathbf{x}(100)=\overline{\mathbf{t}}$.

(g) $\mathbf{x}(101)=10 \overline{\mathbf{t}}$.

(h) $\mathbf{x}(110)=1 \overline{\mathbf{t}}$.

(i) $\mathbf{x}(0010)=001001 \overline{\mathbf{t}}$.

(j) $\mathbf{x}(1101)=110 \overline{\mathbf{t}}$. 


\section{Proof.}

(a) We note that $\mathbf{t}$ is an overlap-free sequence beginning with 1 . On the other hand, the set of overlap-free sequences beginning with a given word is a closed set (a limit of overlapfree sequences is also overlap-free). Let $\mathbf{y}$ be the least overlap-free sequence beginning with 1. Then $\mathbf{y} \leq \overline{\mathbf{t}}$. Hence $\mathbf{y}$ must start with 1001 . Now, from Lemma 3 there exists an infinite sequence $\mathbf{x}$ such that $\mathbf{y}=\mu(\mathbf{x})$. The sequence $\mathbf{x}$ is overlap-free from assertion 2 (a) in Lemma 2. As $\mathbf{x}$ begins with 1 , we have $\mathbf{y} \leq \mathbf{x}$. Hence $\mu(\mathbf{y}) \leq \mu(\mathbf{x})=\mathbf{y}$, since $\mu$ is order-preserving. The sequence $\mu(\mathbf{y})$ is overlap-free as it is the image under $\mu$ of an overlap-free sequence. Hence we must have $\mu(\mathbf{y})=\mathbf{y}$ from the minimality of $\mathbf{y}$. Hence $\mathbf{y}=\overline{\mathbf{t}}$. [We remark that this result was already proved by Berstel [4] in the following "dual" form: the lexicographically greatest infinite overlap-free word on the binary alphabet $\{0,1\}$ that begins with 0 is the Thue-Morse sequence $\mathbf{t}=01101001 \cdots$.

(b) Suppose that $\mathbf{x}(w)$ exists, and that $z \mathbf{x}(w)$ is overlap-free. Hence $z \mathbf{x}(w)$ is an overlapfree sequence beginning with $z w$. Let $z \mathbf{y}$ be an infinite overlap-free sequence beginning with $z w$, that satisfies $z \mathbf{y} \leq z \mathbf{x}(w)$. Then $\mathbf{y}$ is an overlap-free sequence beginning with $w$, and that satisfies $\mathbf{y} \leq \mathbf{x}(w)$. Hence $\mathbf{y}=\mathbf{x}(w)$, and $z \mathbf{y}=z \mathbf{x}(w)$.

Suppose now that $\mathbf{x}(w)$ exists, and that $w w_{1}$ is a prefix of $\mathbf{x}(w)$. Suppose that $\mathbf{y}$ is an overlap-free sequence that begins with $w w_{1}$, and that $\mathbf{y} \leq \mathbf{x}(w)$. Since $\mathbf{y}$ also begins with $w$, we must have $\mathbf{y}=\mathbf{x}(w)$.

(c) We note that an overlap-free sequence beginning with 001 cannot be smaller than $00100 \cdots$, hence (no overlap) than $0010011 \cdots$. Now $001001 \overline{\mathbf{t}}$ is overlap-free from assertion 4 of Lemma 2. It then suffices to note that, from the first assertion in (b) above, $001001 \overline{\mathbf{t}}$ is the lexicographically least sequence beginning with 0010011. Hence $\mathbf{x}(001)=\mathbf{x}(0010011)=$ $001001 \overline{\mathbf{t}}$. Similarly an overlap-free sequence beginning with 0 or 00 cannot be smaller than $00100 \cdots$, and the same reasoning applies: hence $\mathbf{x}(0)=\mathbf{x}(00)=001001 \overline{\mathbf{t}}$.

(d) to (j) Using properties 3 and 4 of Lemma 2, and assertions (a), (b) and (c) above, we have:

$$
\begin{aligned}
& \mathbf{x}(010)=0 \overline{\mathbf{t}}, \text { since } \mathbf{x}(01)=0 \overline{\mathbf{t}} \\
& \mathbf{x}(011)=01 \overline{\mathbf{t}} ; \\
& \mathbf{x}(100)=\overline{\mathbf{t}}, \text { since } \mathbf{x}(1)=\overline{\mathbf{t}} \\
& \mathbf{x}(101)=10 \overline{\mathbf{t}} ; \\
& \mathbf{x}(110)=1 \overline{\mathbf{t}}, \text { since } \mathbf{x}(10)=\overline{\mathbf{t}} ; \\
& \mathbf{x}(0010)=001001 \overline{\mathbf{t}}, \text { since } \mathbf{x}(001)=001001 \overline{\mathbf{t}} \\
& \mathbf{x}(1101)=110 \overline{\mathbf{t}}, \text { since } \mathbf{x}(110)=1 \overline{\mathbf{t}} .
\end{aligned}
$$

We are now ready to state and prove our main theorem.

Theorem 1 Let $w$ be a finite binary word such that there exists an infinite overlap-free binary sequence which begins with $w$. Let $\mathbf{x}$ be the lexicographically least infinite overlap-free binary sequence which begins with $w$. Then there exists a suffix of $\mathbf{x}$ that is equal to a suffix of the Thue-Morse sequence $\overline{\mathbf{t}}=10010110 \cdots$. The construction of this minimal sequence is given by an explicit algorithm. 
Proof. We will prove the result by induction on the length of $w$. The induction will show the construction to be algorithmic.

Using Proposition 1 above, we see that the result has already been obtained if the word $w$ has length at most 3 or is equal to 0010 or 1101 . The result is also true if $w=00100$ and $w=001001$, using Proposition $1(\mathrm{~b})$, and the equality $\mathbf{x}(0010)=001001 \overline{\mathbf{t}}$ : this gives $\mathbf{x}(00100)=\mathbf{x}(001001)=001001 \overline{\mathbf{t}}$. Furthermore the result holds also true if $w=11011$ or $w=110110$, since we obtain, using Lemma 2 (e) and Proposition 1 (i) and (b), that $\mathbf{x}(11011)=\mathbf{x}(110110)=110110010110 \overline{\mathbf{t}}$.

If $w$ has length $\geq 4$, and is not one of the six words above, we will prove that $\mathbf{x}(w)$ ends with $\mu\left(\mathbf{x}\left(w^{\prime}\right)\right)$ for some $w^{\prime}$ such that $\left|w^{\prime}\right|<|w|$. Since $\mu(\overline{\mathbf{t}})=\overline{\mathbf{t}}$, the image by $\mu$ of an infinite word having a suffix in common with $\overline{\mathbf{t}}$ has the same property: hence the induction hypothesis for $w^{\prime}$ will imply the required result for $w$.

Using Lemma 3, write $w=u \mu(y) v$. Note that, from the proof of the second part of Lemma 3, the word $v$ is either empty or has length 1 . Furthermore $\mathbf{x}(w)$ has to begin with $u \mu(y) v \bar{v}=w \bar{v}$, hence $\mathbf{x}(w)=\mathbf{x}(w \bar{v})$.

- If $u$ is empty, then $\mathbf{x}(w)=\mathbf{x}(w \bar{v})=\mathbf{x}(\mu(y v))=\mu(\mathbf{x}(y v))$. We have $|y v|<|\mu(y) v|=|w|$, since $y$ cannot be empty $(|w| \geq 4)$.

- If $u=0$, using Lemma 2 and Proposition 1 we have $1 \mathbf{x}(w)=1 \mathbf{x}(w \bar{v})=1 \mathbf{x}(0 \mu(y v))=$ $\mathbf{x}(10 \mu(y v))=\mathbf{x}(\mu(1 y v))=\mu(\mathbf{x}(1 y v))$. We have $|1 y v|<|0 \mu(y) v|=|w|$, since $y$ cannot be empty. The same reasoning holds for $u=1$.

- If $u=00$, we have $\mathbf{x}(w)=\mathbf{x}(w \bar{v})=\mathbf{x}(00 \mu(y v))$. Let $\mathbf{x}(w)=00 \mu(y v) \mu(\mathbf{z})$. Using Lemma 2, we know that $1 y v(\mathbf{z})$ has to be overlap-free, and that $y v(\mathbf{z})$ has to begin with 101.

- If $4 \leq|w| \leq 6$, the equality $w=00 \mu(y) v$ easily implies that $w$ is one of the words 0010 , 00100,00101 , or 001001 . The cases $w \in\{0010,00100,001001\}$ have been excluded. The case $w=00101$ is impossible: namely $\mathbf{x}(00101)=\mathbf{x}(001011)$, since 001010 contains an overlap. But the word 001011 is not of the form $00 \mu(y) v$ with $|v| \leq 1$.

- If $|w| \geq 7$, then the equality $w=00 \mu(y) v$ shows that $|y v| \geq 3$, hence $y v$ has to begin with 101. Let $y v=101 y^{\prime}$. We thus have $\mathbf{x}(00 \mu(y v))=\mathbf{x}\left(00 \mu\left(101 y^{\prime}\right)\right)=00 \mu\left(101 y^{\prime} \mathbf{z}\right)$. Then, by Lemma $2,1\left(101 y^{\prime} \mathbf{z}\right)=\mathbf{x}\left(1101 y^{\prime}\right)$. Finally $\left|1101 y^{\prime}\right|=|1 y v|<|00 \mu(y) v|=|w|$.

The same reasoning holds if $u=11$.

\section{Remark 1.}

The algorithm we give resembles an algorithm given in [11] to decide (in linear time) whether a finite binary word contains an overlap.

\section{Remark 2.}

- Let us show an example of how the algorithm works. This example also shows that it is possible for the lexicographically least sequence beginning with a word $w$ to have no suffix equal to the Thue-Morse sequence $\overline{\mathbf{t}}$. Let $w=0010110$. If there exists an infinite overlap-free 
sequence $\mathbf{s}$ beginning with $w$, say $\mathbf{s}=w \mathbf{x}$, then its factorization must be $\mathbf{s}=0 \mu(001 \mathbf{y})$. Now $\mathbf{s}$ is the least overlap-free sequence beginning with $w$ if and only if $1001 \mathbf{y}$ is the least overlapfree sequence beginning with 1001 (assertion 2 (b) in Lemma 2). Now the factorization of $1001 \mathbf{y}$ must be $\mu(10 \mathbf{z})$, with $\mathbf{y}=\mu(\mathbf{z})$. And $1001 \mathbf{y}$ is the least overlap-free sequence beginning with 1001 if and only if $10 \mathbf{z}$ is the least overlap-free sequence beginning with 10. From Proposition 1, we see that the least overlap-free sequence beginning with 100 is $\overline{\mathbf{t}}$, and that the least overlap-free sequence beginning with 101 is $10 \overline{\mathbf{t}}$. Hence the least overlap-free sequence beginning with 10 is $\mathbf{t}$. Hence $10 \mathbf{z}=\overline{\mathbf{t}}$. We thus have successively $1001 \mathbf{y}=1001 \mu(\mathbf{z})=\mu(10 \mathbf{z})=\mu(\overline{\mathbf{t}})=\overline{\mathbf{t}}$. Then $1 \mathbf{s}=10 \mu(001 \mathbf{y})=\mu(1001 \mathbf{y})=\mu(\overline{\mathbf{t}})=\overline{\mathbf{t}}$. Hence $\mathbf{s}$ is the sequence obtained by deleting the first letter of $\overline{\mathbf{t}}$.

Finally, we give as a corollary of the above result a new proof of a result obtained in $[1,3]$ in relation with iterations of continuous functions and kneading sequences.

Corollary 1 For a binary sequence $\mathbf{s}$ let $S(\mathbf{s})$ be the sequence obtained by deleting the first letter of $\mathbf{s}\left(S\right.$ is also called the shift). Let $S^{k}$ be the $k$-th iterate of the map $S$. Let $\mathbf{a}=S(\mathbf{t})=$ $11010011001 \cdots$. Then, for each $k \geq 1$, we have $\overline{\mathbf{a}}<S^{k}(\mathbf{a})<\mathbf{a}$.

Proof. Since the sequence $\mathbf{t}$ is overlap-free, so is the sequence $\mathbf{a}$. Hence a cannot be periodic. This implies that $S^{k}(\mathbf{a})$ cannot be equal to either a or $\overline{\mathbf{a}}$ for $k \geq 1$. We thus have only to prove that, for all $k \geq 1$ (actually this is true for all $k \geq 0$ ), $\overline{\mathbf{a}} \leq S^{k}(\mathbf{a}) \leq \mathbf{a}$. We first prove that, for each $k \geq 0$, we have $\overline{\mathbf{a}} \leq S^{k}(\mathbf{a})$. Since $\overline{\mathbf{a}}=0010110 \cdots$ we are done if $S^{k}(\mathbf{a})$ begins with 1 or with 01 or with 0011. If $S^{k}(\mathbf{a})$ begins with 0010, it cannot begin with 00100. For if this were the case, $S^{k}(\mathbf{a})$ would begin with 001001 since it is overlap-free. But the word 001001 is not extendable to the left into an overlap-free word, hence cannot occur in the sequence $\mathbf{a}$, since this sequence is extendable to the left into $\mathbf{t}$ which is overlap-free.

Hence $S^{k}(\mathbf{a})$ begins with 00101, hence with 001011, hence with 0010110. But, as shown by the remark above, the least overlap-free sequence beginning with 0010110 is $S(\overline{\mathbf{t}})=\overline{\mathbf{a}}$. Hence $S^{k}(\mathbf{a}) \geq \overline{\mathbf{a}}$.

We now prove that, for each $k \geq 0$, we have $S^{k}(\mathbf{a}) \leq \mathbf{a}$. Since $\mathbf{a}=11010011 \cdots$, we are done if $S^{k}(\mathbf{a})$ begins with 0, 10, or 1100. Hence we can suppose that $S^{k}(\mathbf{a})$ begins with 1101.

It is easy to see that $S^{k}(\mathbf{a})$ cannot begin with 11011 because the only substrings of $\mathbf{t}$ starting at even positions are 10 and 01 . Hence $S^{k}(\mathbf{a})$ begins with 11010, hence with 110100, hence with 1101001. But the "dual" of the above remark shows that the lexicographically greatest overlap-free sequence beginning with 110100110 is $S(\mathbf{t})=\mathbf{a}$ and the result is proved.

\section{Remark 3.}

An even simpler argument shows that the sequence $\mathbf{b}=001001 \overline{\mathbf{t}}$ satisfies, for all $k \geq 0$, $\mathbf{b} \leq S^{k}(\mathbf{b}) \leq \overline{\mathbf{b}}$.

We give another simple corollary.

Corollary 2 The lexicographically least overlap-free sequence that is extendable to the left to a doubly-infinite overlap-free sequence is $S(\overline{\mathbf{t}})$. 
Proof. We have seen that the lexicographically least sequence on $\{0,1\}$ is the sequence $001001 \overline{\mathbf{t}}$. This sequence cannot be extended to the left to an overlap-free sequence, since concatenating either 0 or 1 to the front creates an overlap. Now let us consider the sequence $S(\overline{\mathbf{t}})$. It begins with 0010110 and is the least overlap-free sequence having this prefix from Remark 2 above. It is not possible to find a smaller sequence that is extendable to the left to an overlap-free doubly-infinite sequence, since the prefix 00100 is forbidden from the claim above. Hence such a sequence must begin with 00101, hence with 0010110.

On the other hand, it is well-known that $\overline{\mathbf{t}}$ is extendable to the left to a doubly-infinite overlap-free sequence ([15, Satz 7], [12], [5, p. 30]).

\section{The lexicographically least square-free sequence}

As already proved by Thue, it is possible to construct, on a three-letter alphabet, a sequence without squares, i.e., without any factor of the form $w w$, where $w$ is a nonempty word. (It is readily checked that there is no square-free word of length $\geq 4$, and hence no square-free infinite word, on a two-letter alphabet.) Now take the alphabet to be $\{0,1,2\}$. What is the lexicographically least square-free sequence over $\{0,1,2\}$ ? We do not know a simple description of this sequence. Using the results of Shelton $[13,8]$ it can be proved that the first twenty terms are

$$
01020120210120102012 .
$$

In particular, is it true that this sequence can be generated by a finite automaton (in the sense of [7]; see also $[2,9])$ ? As proved above the lexicographically least overlap-free sequence over $\{0,1\}$ is $001001 \overline{\mathbf{t}}$, and hence is 2 -automatic.

\section{Acknowledgments}

We thank the referee for many useful remarks, particularly those concerning our proof of the main theorem. We also thank Larry Cummings, who read a draft of this paper and made many valuable suggestions. The first two authors acknowledge with thanks the hospitality of the University of Waterloo, where this paper was largely written. 


\section{References}

[1] J.-P. Allouche, Théorie des nombres et automates, Thèse d'État, Université Bordeaux I, 1983.

[2] J.-P. Allouche, Automates finis en théorie des nombres, Exposition. Math. 5 (1987), 239-266.

[3] J.-P. Allouche and M. Cosnard, Itérations de fonctions unimodales et suites engendrées par automates, C. R. Acad. Sci. Paris, Sér. A 296 (1983), 159-162.

[4] J. Berstel, A rewriting of Fife's theorem about overlap-free words, in J. Karhumäki, H. Maurer, G. Rozenberg, eds., Results and Trends in Theoretical Computer Science, Lecture Notes in Computer Science 812, Springer-Verlag, 1994, pp. 19-29.

[5] J. Berstel, Axel Thue's papers on repetitions in words: a translation, Publications du Laboratoire de Combinatoire et d'Informatique Mathématique, Université du Québec à Montréal 20, 1995.

[6] J. Berstel, P. Séébold, A characterization of overlap-free morphisms, Disc. Appl. Math. 46 (1993), 275-281.

[7] A. Cobham, Uniform tag sequences, Math. Systems Theory 6 (1972), 164-192.

[8] J. D. Currie, On the structure and extendibility of k-power free words, Eur. J. Comb. (1995) 16, 111-124.

[9] F. M. Dekking, M. Mendès France, and A. van der Poorten, Folds!, Math. Intelligencer 4 (1982), 130-138, 173-181, 190-195.

[10] Y. Kobayashi, Repetition-free words, Theoret. Comput. Sci. 44 (1986), 175-197.

[11] A. J. Kfoury, A linear-time algorithm to decide whether a binary word contains an overlap, RAIRO Inform. Théor. App. 22 (1988), 135-145.

[12] M. Morse and G. A. Hedlund, Unending chess, symbolic dynamics and a problem in semigroups, Duke Math. J. 11 (1944), 1-7.

[13] R. O. Shelton (I, II, III) and R. P. Soni (II, III), Aperiodic words on three symbols I, II, III, J. Reine Angew. Math. 321; 327; 330 (1981), 195-209; 1-11; 44-52.

[14] A. Thue, Über unendliche Zeichenreihen, Norske vid. Selsk. Skr. Mat. Nat. Kl. 7 (1906), 1-22. Reprinted in T. Nagell, ed., Selected Mathematical Papers of Axel Thue, Universitetsforlaget, Oslo, 1977, pp. 139-158.

[15] A. Thue, Über die gegenseitige Lage gleicher Teile gewisser Zeichenreihen, Norske vid. Selsk. Skr. Mat. Nat. Kl. 1 (1912), 1-67. Reprinted in T. Nagell, ed., Selected Mathematical Papers of Axel Thue, Universitetsforlaget, Oslo, 1977, pp. 413-478. 